\title{
Probing of polymer to carbon nanotube surface interactions within highly aligned electrospun nanofibers for advanced composites
}

\author{
Simon G. King ${ }^{\mathrm{a}, \mathrm{b}}$, Nicholas J. Terrill ${ }^{\mathrm{c}}$, Andrew J. Goodwin ${ }^{\mathrm{b}}$, Robert Stevens ${ }^{\mathrm{d}}$, Vlad Stolojan ${ }^{\mathrm{a}}$, S.Ravi P. Silva ${ }^{\mathrm{a}, *}$ \\ advanced Technology Institute, University of Surrey, Guildford, Surrey GU2 7XH, UK \\ b Thomas Swan \& Co. Ltd., Rotary Way, Consett, County Durham, DH8 7ND, UK \\ ${ }^{c}$ Diamond Light Source Ltd, Diamond House, Harwell Science and Innovation Campus, Didcot Oxfordshire OX11 ODE, UK \\ ${ }^{\mathrm{d}}$ Nottingham Trent University, Nottingham, NG1 4BU, UK
}

Article history:

Received 3 May 2018

Received in revised form 6 June 2018

Accepted 8 June 2018

Available online $\mathrm{xxx}$

\begin{abstract}
By electrospinning poly(ethylene oxide) (PEO)-blended sodium dodecyl sulfate (SDS) functionalized carbon nanotube (CNT) solutions, we engineered single- and double-walled nanotubes into highly aligned arrays. CNT alignment was measured using electron microscopy and polarised Raman spectroscopy. Mechanical tensile testing demonstrates that a CNT loading of $3.9 \mathrm{wt} \%$ increases the ultimate tensile strength and ductility of our composites by over a factor of 3 , and the Young's modulus by over a factor of 4 , to $\sim 260 \mathrm{MPa}$. Transmission electron microscopy (TEM) reveals how the aligned nanotubes provide a solid structure, preventing polymer chains from slipping, as well as polymer crystallisation structures such as 'shish-kebabs' forming, which are responsible for the improved mechanical properties of the composite. Differential scanning calorimetry (DSC) and small angle X-ray scattering (SAXS) reveals micellar and hexagonal columnar structures along the axis of the fibers, some of which are associated with the presence of the CNT, where these hexagonal structures are associated with the SDS functionalization on the CNT surfaces. This work demonstrates the benefits of CNT alignment within composites, revealing the effectiveness of the electrospinning technique, which enables significantly improved functionality, increasing the utility of the composites for use in many different technological areas.
\end{abstract}

(C) 2018 .

\section{Introduction}

Carbon nanotubes (CNT) are of interest due to their outstanding mechanical, thermal and electrical properties, combined with their high aspect ratio: nano-sized diameters and typically microns in length [1]. It has been well documented that, due to the difficulty in growing continuous CNT material to macroscopic lengths [2], an easier route to harnessing their unique properties on a larger scale is to align and connect or combine the individual tubes [3]. A high degree of CNT alignment will result in the magnification of the uniaxially excellent properties of individual nanotubes to macroscopic length-scales. This scalable alignment technology therefore is a strong candidate for large-area manufacturing of highly-conductive light-weight CNT yarns/wires [4] and advanced composite materials [5]. A secondary challenge is to ensure a good, strong chemical bond between the matrix and the CNT content, to enable both the transfer of mechanical strain but also to improve electrical and thermal conductivity between the matrix and the CNT [6]. Although there are several successful methods of CNT alignment [7], one simple and elegant method is electrospinning, which forms nanofibers based on the uniaxial stretching of a viscoelastic solution, utilising electrostatic forces to extend the solution as it solidifies, before collection $[8,9]$. Furthermore, the stretching and shear flow occurring during the travel of the

\footnotetext{
* Corresponding author.

Email address: s.silva@surrey.ac.uk (S.RaviP. Silva)
}

electrospun polymer from the source to the collector can lead to controlled crystallisation of the polymer chains [10]. When they are introduced, CNT incorporation has been observed to promote crystallisation along their surfaces as a regular structure known as 'shish-kebab' [11], where the CNT acts as the central directional nucleation point, which promotes the polymer to form spherulites along the nanotubes length, resembling similarity of meat layers along a kebab stick. This crystalline formation facilities load transfer from the polymer to the embedded nanotubes, whilst improving the electrical and thermal conductivities of the resulting composite.

Electrospinning (see Fig. 1, diagram A) has been commonly used for nanofiber manufacture since its inception in 1887 [12], with modern utilisation of the nanofibers covering a vast range of applications, such as polymer textiles, air filters, tissue-growth substrates, etc. [13]. Various publications report on how electrospinning CNT loaded solutions, dispersed within a suitable polymer, can be used to produce arrays of aligned fibers, containing the embedded, aligned nanotubes [9,14-17]. These CNT-embedded polymer nanofibers exhibit improved properties, depending on the polymer and type of CNT reinforcement, but few studies explore the polymer/CNT interactions which result in the overall material enhancement. By using high-quality, long nanotubes $(>1 \mu \mathrm{m})$ functionalized with sodium dodecyl sulfate (SDS), the electrospinning process was used to align nanotubes within poly(ethylene oxide) (PEO) nanofibers (a water-soluble polymer), producing a nano-composite material. Furthermore, we will demonstrate that not only are the electrospun fibers well-aligned, but 

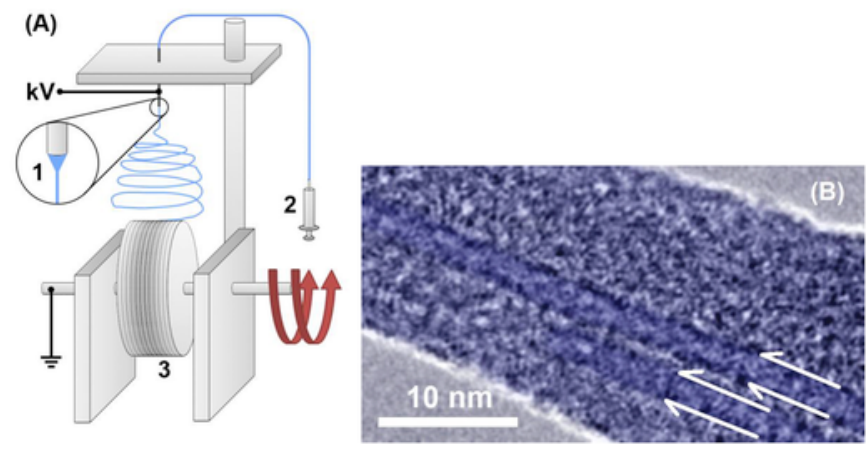

Fig. 1. (A) A schematic diagram of the electrospinning setup used, showing 1) the single needle spinneret, 2) a pumped syringe and 3) the grounded high-speed collector. (B) A false-colour transmission electron micrograph of the SDS-coated CNT material embedded within the PEO electrospun nanofiber. This image demonstrates how the electrospinning technique aligns the CNT within the polymer nanofiber (direction of CNT walls arrowed). (A colour version of this figure can be viewed online.)

also that the CNT content is also well aligned. The inclusion of the highly-aligned nanotubes within the PEO was found to enhance the mechanical properties of the material by a significant factor, increasing the Young's modulus by up to $430 \%$. This we attribute to be the result of the CNT functionalization promoting the growth of polymer nano-crystalline structures in their vicinity, due to the shear flow and stretching experienced during the electrospinning process [18]. Thermal analysis revealed that although the CNT presence lowered the overall crystal content as well as the melting temperature, it promoted crystal growth and initiated crystallisation at a higher temperature on cooling. This change in crystallisation behaviour was evidenced by electron microscopy and X-ray scattering exposing how although the $\mathrm{CNT}$ presence lowers overall crystalline content, they equally promote the growth of an alternative crystalline structure, oriented with the nanofibers, as a rigid support for the polymer chains, allowing for the efficient transfer of mechanical stress from the polymer matrix to the CNT loading.

We analyse the CNT distribution inside the polymer fibers, their degree of alignment and reveal the polymer crystallisation around the nanotubes using: Raman spectroscopy to assess CNT quality and alignment, scanning transmission electron microscopy (STEM) to image CNT interaction and positioning within the nanofibers, differential scanning calorimetry (DSC) to probe crystallinity, and small-an- gle X-ray scattering (SAXS) to evaluate nanomorphology. In particular, SAXS is able to look at macroscopic-sized materials, as opposed to inferring polymer crystallisation from individual nanotubes in single polymer fibers $[18,19]$. We assess the mechanical properties of the composite using tensile testing, for different CNT loadings.

\section{Experimental section}

PEO supplied by Sigma Aldrich (Merck) was chosen as the spinnable polymer in this study, with an average molecular weight of $\sim 2,000,000 \mathrm{M}_{\mathrm{v}}$. It provided the necessary viscoelastic properties required for electrospinning, while being low-cost and water-soluble. The single/double-wall CNT material was manufactured by Thomas Swan \& Co. Ltd. using a chemical vapour deposition (CVD) process [20], and supplied as their Elicarb ${ }^{\circledR}$ 'wet-cake' product (3.7\% wt. CNT in an aqueous solution, forming a (caviar'). Raman analysis revealed that the CNT wet-cake contained single- and double-walled with predominant diameters of $1.23 \mathrm{~nm}$ and $1.07 \mathrm{~nm}( \pm 0.05 \mathrm{~nm})$ for the single-walled tubes, and $1.61 \mathrm{~nm}( \pm 0.05 \mathrm{~nm})$ for the double-walled tubes (see Supplementary Information Fig. S1). The CNT content was dispersed with sodium dodecyl sulphate (SDS) at a weight-ratio of 1:10, CNT to SDS respectively, previously reported as being the ideal ratio of surfactant to CNT $[21,22]$. Two aqueous solutions were mixed, containing $0.004 \%$ and $0.1 \%$ weight CNT material. These dispersions were blended with PEO after the CNT dispersion, so as to prevent the ultra-sonic irradiation destroying the polymer chains, which would render the solution unsuitable for electrospinning [23]. After all the solvent evaporates during and after electrospinning, these solutions will effectively produce PEO nanofibers containing a CNT content of $0.7 \%$ and $3.9 \%$ weight respectively. Fig. 1, image B (bright-field transmission image taken on a Hitachi HD-2300A STEM), illustrates how the embedded CNT material has been pulled into alignment by the whipping/drawing effect exerted on the polymer during the electrospinning process. This drawing/stretching effect not only pulls the polymer chains and subsequently the CNT loading into alignment, but also reduces the diameter of the electrospun fibre, so that it is much smaller than the length of the CNT, forcing it to align.

In some situations, such as tensile testing and SAXS, it was necessary to first roll the aligned CNT nanofibers into ropes or yarns. This was done by hand, rolling the nanofibers parallel to the direction of the fibre, producing a rope with diameters ranging from 200 to $300 \mu$ $\mathrm{m}$, comprised of thousands of nanofibers (see Fig. 2). Cross-sectional

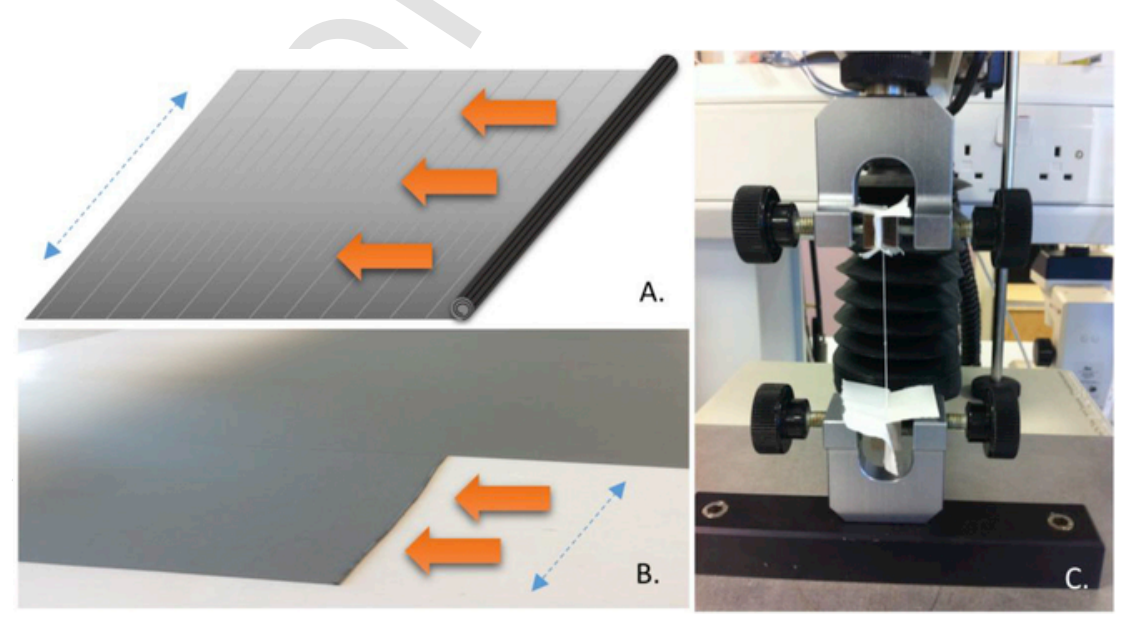

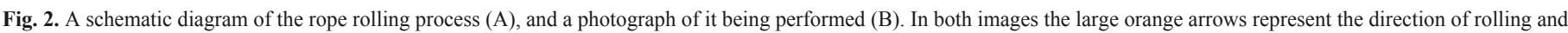

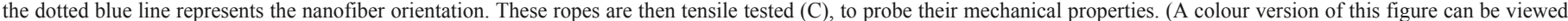
online.) 
area of these ropes was measured using both a micrometre gauge and a high-powered optical microscope.

Polarised Raman spectroscopy was conducted using a Renishaw micro-Raman spectrometer equipped with a $785 \mathrm{~nm}$ (red) laser. Before measuring the samples, the laser's plane of polarisation, and the spectrometers grating calibration were confirmed using a silicon reference sample. For polarised Raman analysis on the rolled CNT ropes, the incident laser polariser was fixed at the laser's measured plane of polarisation and the specimen rotated, enabling measurements to be taken with the laser polarisation both parallel and perpendicular to the nanofiber axis.

To conduct mechanical testing, the nanofiber ropes were secured into a 'TA XT Plus Texture Analyser' using the clamps provided. Each sample was analysed using a test rope length of $4.0 \pm 0.1 \mathrm{~cm}$. Each test was conducted at a recorded room temperature of $21^{\circ} \mathrm{C}$, at a testing speed of $0.5 \mathrm{~mm} / \mathrm{s}$, where measurements were taken 50 times a second.

DSC was used to probe crystallinity of the various nanofiber samples, this was conducted on a TA Instruments Q1000 DSC. Each sample of approximately $5 \mathrm{mg}$ was tested using a heat-cool-heat method from $0{ }^{\circ} \mathrm{C}$ to $100^{\circ} \mathrm{C}$ at $10^{\circ} \mathrm{C} / \mathrm{min}$. This allowed for the initial crystallinity of the as-spun nanofibers to be compared to a sample if it was simply blended and set, giving an indication of any crystal structures produced during the electrospinning process.

SAXS was performed on I22 beamline at the Diamond Light Source, located at the Harwell Science and Innovation Campus in Oxfordshire, in the UK. All SAXS equipment set-up and technical specifications can be found on their website [24]. Before conducting any SAXS analysis, the X-ray detector was calibrated for the scattering vector ' $q$ ' using wet rat-tail collagen, which provides a primary spacing $\mathrm{d}=670 \AA[25,26]$, thus ensuring accurate measurements for low ' $\mathrm{q}$ ' experiments. To maximise X-ray scattering and characterise the sample, the fibers were analysed as a rolled rope (see Fig. 2). The aligned nanofiber ropes were positioned horizontally in the sample housing, where the X-ray beam was scanned across the sample, perpendicular to the fibre axis, up to down. Diffraction images were taken at each $0.1 \mathrm{~mm}$ step across the sample, which were then plotted by taking an intensity sector integration as a function of q value.

\section{Results and discussion}

To verify the CNT alignment embedded within the nanofibers, the samples were first characterised by polarised Raman spectroscopy. Carbon nanotubes have a variety of specific Raman spectroscopic peaks, corresponding to different vibrational modes. One such peak is the radial breathing mode (RBM), which corresponds to the expansion and contraction of the single walled tube radially and is unique to single or few walled nanotubes (see Supplementary Fig. S1). Hwang et al. (2000) [27] showed that, by recording the Raman intensity of the CNT RBM signal as a function of angle to the Raman laser's polarisation direction, a measure of the degree of CNT alignment within a polymer fibre can be obtained. For perfectly aligned nanotubes, the intensity of the RBM peak is proportional to $\cos ^{4} \theta$, where $\theta$ is the angle between the laser polarisation and the fibre alignment axis. Fig. 3 shows the measured intensities for the RBM of a $1.23 \mathrm{~nm}$ single-walled CNT $\left(202 \mathrm{~cm}^{-1}\right)$ peak as a function of angle between the laser polarisation direction and that of the aligned PEO/ CNT nanofibers. At each angle several locations were scanned, to average over local variations in CNT orientation. The data was fitted using a $\cos ^{4} \theta$ function, which allowed for direct comparison to the theory. It was found that the PEO polymer was depolarising the Raman excitation laser, obscuring the results. To overcome this, the polymer was removed by evaporation within an inert atmosphere by heating the sample to $450{ }^{\circ} \mathrm{C}$ (based on TGA analysis), where further

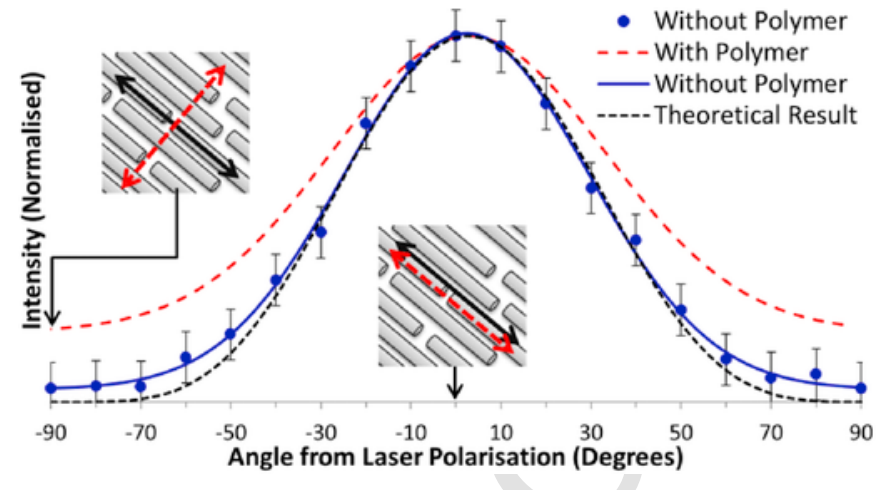

Fig. 3. The measured radial breathing mode intensities $(202 \mathrm{~cm}-1)$ as a function of fibre angle $\boldsymbol{\theta}$ (degrees) for PEO nanofibers embedded with $3.9 \%$ CNT. The solid line represents a plotted $\cos ^{4} \theta$ line of best fit from the measured data, while the dotted line represents $\boldsymbol{y}=\cos ^{4} \boldsymbol{\theta}$, which is the theoretical result for perfectly aligned nanotubes. The error bars represent the largest standard error in the mean across all data points. The two small schematic diagrams at $-90 / 90^{\circ}$ and $0^{\circ}$, demonstrate the CNT oriention (solid arrow) compared to the plane of polarisation of the laser (dotted arrow). (A colour version of this figure can be viewed online.)

polarised Raman (see Fig. 3, Without Polymer) showed a much narrower distribution. The measured data taken without the polymer followed the theoretical result for perfectly aligned nanotubes, which confirms the high degree of CNT alignment within the sample.

The effects of CNT loading in composites has already been widely explored, repeatedly revealing how CNT loading, especially when aligned, can result in a varying degree of mechanical improvements [28-30]. These improvements have been reported to be the result of induced crystallisation, with the CNT as the nucleation point. This effect is enhanced further by the electrospinning process, where the rapid drawing and stretching of the polymer, results in stress-induced crystallisation [31,32]. To reduce or avoid damage by the tensometer clamps, the electrospun PEO/CNT nanofibers were tested as rolled ropes (see Fig. 2). Care was taken to minimise the twist of the fibers around the axis of the rope; any twist in the ropes would obscure the mechanical performance of the sample through untwisting and this would also lead to residual stresses, which would encourage material failure and obscure results. The ropes were tested as outlined in the experimental section and the stress/strain curves for each of the sample are shown in Fig. 4; tests were considered valid if the fibre failed near the middle of the rope, rather than at the mechanical clamps.

The CNT introduction into the nanofibers drastically improves the mechanical performance of the material, increasing the ultimate tensile strength by up to $320 \%$ and increasing the ductility by up to $315 \%$ (from the measured 100\% PEO samples). All the samples successfully fractured in the centre of the rope, with some samples showing individual fibers failing (or fraying) without the complete failure of the sample, Fig. 4 arrow B is an example. Samples that didn't fail at the centre were retested, to ensure that the failure wasn't caused by an accidently introduced failure mechanic such as tears or twists during handling. From these plots, it was possible to measure the Young's modulus of each rope. The CNT loading within the PEO nanofiber increased the Young's modulus by up to $430 \%$, from $60 \pm 1 \mathrm{MPa}$ to $257 \pm 1 \mathrm{MPa}$. These results are a significant improvement on previous $\mathrm{PEO} / \mathrm{CNT}$ composites tested [34]. This we believe, is due to the CNT alignment, as opposed to randomly dispersed through the PEO, as well as because the nanotubes used in this study had a low defect density and high linearity, as evidenced in TEM images (such as shown in Fig. $1 \mathrm{~B})$.

The shape of the stress-strain curves revealed that all samples experienced a degree of strain-hardening. However, as CNT loading 


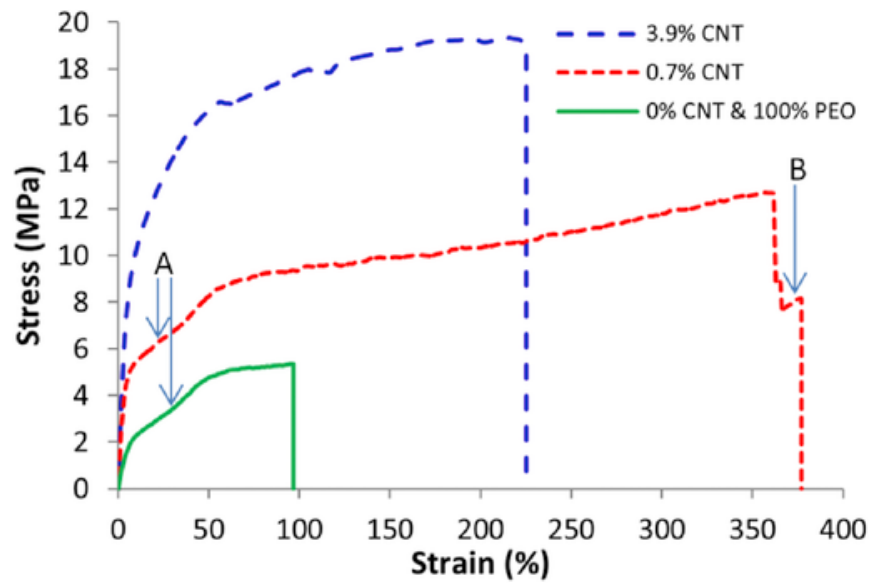

Fig. 4. The stress to failure results for PEO nanofiber ropes, with a CNT loading of $0 \%$, $0.7 \%$ and $3.9 \%$ weight. The measured corresponding Young's Moduli were found to be $60 \pm 2 \mathrm{MPa}, 182 \pm 5 \mathrm{MPa}$ and $257 \pm 8 \mathrm{MPa}$, respectively. The slight inflection point, found just after the elastic region (arrowed as A), corresponds to polymer heating as a result of extension-induced crystallisation [33]. The two-stage failure found just before the end of the $0.7 \%$ CNT loading test (arrowed as B), corresponds to many fibers in the rope failing, but with the remaining fibers briefly continuing to hold before failing themselves. (A colour version of this figure can be viewed online.)

was increased so did the degree of hardening experienced by the polymer, leading to greater strength performances before the ropes failure. Compared to the other two curves, the $3.9 \%$ CNT sample displayed a slightly different shape. This was due to the reinforcement provided by the CNT loading, where although it continued to improve the strength of the material, it also reduced ductility when compared to the $0.7 \%$ CNT sample. This is due to the high-strength brittle properties of the increased CNT loading begin to dominate the mechanical response.

PEO as an extruded macroscopic block has been documented to display Young's moduli from 5 to $9 \mathrm{GPa}$ [35], whereas, when formed into nanofibers has been documented to have a Young's modulus between $45 \mathrm{MPa}$ and 7.6 GPa [36-38]. This reduction in mechanical performance, when formed into a nanofiber, is caused by electrospinning, which modifies the molecular orientation of the polymer chains, reducing the polymer entanglement within the fibre and allowing them to slip when under stress [14]. The large range of mechanical performances reported for electrospun fibers is a result of changing the electrospinning conditions, such as the humidity or the electrical potential, altering the final properties of the material, such as width and alignment etc. This same large variation in mechanical performances can be observed in the CNT samples, and as such all electrospinning conditions were kept constant for this investigation. Although all of the samples tested were manufactured under identical conditions, the only accurate method to compare results with others publications is to tensile test individual fibers under strict controlled conditions [38].

During the tensile testing, it was also found that the polymer stiffened, due to polymer crystallisation under stretching forces [31,32]. Evidence of this can be observed in the $0 \%$ CNT (100\% PEO) plot in Fig. 4 (A), where the curve drops after the elastic response due to heat released as a result of the stress-induced crystallisation [31,32]. It was noticed that, after the CNT-loaded PEO fibers had fractured, the fibre felt very stiff compared to the untested fibers. Additionally, visual inspection revealed that the overall appearance of the rope had become a dark shade of grey as opposed to the original carbon-black. However, this observation was found to be significantly lesser for the PEO only nanofibers, suggesting that the crystallisation was pro- moted by the CNT presence, which was confirmed with DSC analysis, explained later in this report.

TEM images of the CNT loaded nanofibers that were tensile tested revealed how the CNT introduction gives rise to the significant increase in the materials mechanical properties. A micrograph taken of a bundle of nanofibers, which were fixed under tension to a copper TEM grid, is shown in Fig. 5, where image 'A' shows the fibers beginning to neck and fail. However, after a closer look, image ' $\mathrm{B}$ ' revealed how the aligned CNT reinforcements within, are 'holding' the fibers together, providing a solid support structure for the polymer. Another example demonstrating the mechanical enhancement effects provided by the CNT loading can be seen in Fig. 6, where both secondary electron and transmission electron micrographs show the nanotubes bridging the gaps of tears in the polymer nanofibre as it begins to fail, preventing it from fully failing. Although these particular images do not provide any information on the polymer/CNT interface, locating a narrower nanofiber does allow TEM to reveal crystalline nanostructures. Fig. 7, micrograph (A), reveals 'shish-kebab' structures on a small CNT bundle. These structures have been reported to form when a 1D nanomaterial, such as a CNT, acts as a central nucleation point for polymer crystallisation $[11,39,40]$. This growth mechanism allows CNT loading to enhance the mechanical properties by effectively acting as an anchor, providing the polymer chains a 'ridged scaffold' to bind to along the surface along the CNT. This crystalline structure would provide the necessary surface interfaces which would promote the CNT/polymer binding, reducing chain slipping when under stress and giving rise to the mechanical enhancements that we observe in Fig. 4.

Further imaging of larger nanofibers found other crystalline lamella type structures on surface of the fibers. Fig. 7 B highlights these structures found on two nanofibers positions side-by-side, where a two-layer lamella structure can be observed of approximately $5 \mathrm{~nm}$ thick layers. Unlike the other crystal structures observed, these were not a result of the CNT presence or the tensile testing, but rather the electrospinning process and have been reported previously [41]. It is thought that, as the nanofiber is stretched and drawn under the
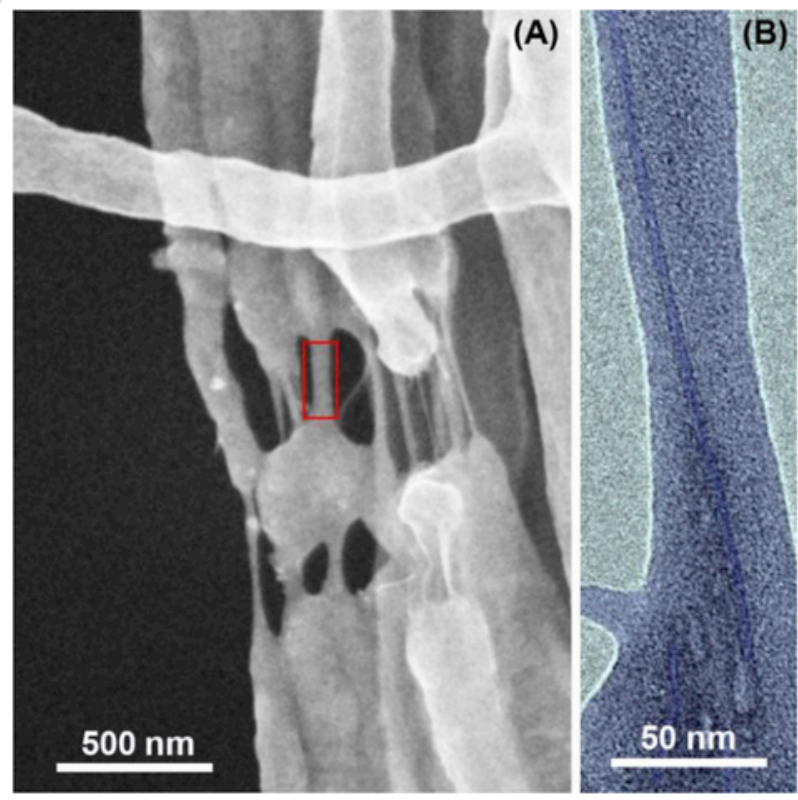

Fig. 5. A secondary electron micrograph of a bundle of CNT loaded PEO nanofibers (A), which have been stretched to breaking point. A closer inspection using transmission electron microscopy (B), reveals the nature of the CNT alignment within the fibers, holding the fibers together, giving rise to the increase in mechanical properties observed during tensile testing. (A colour version of this figure can be viewed online.) 

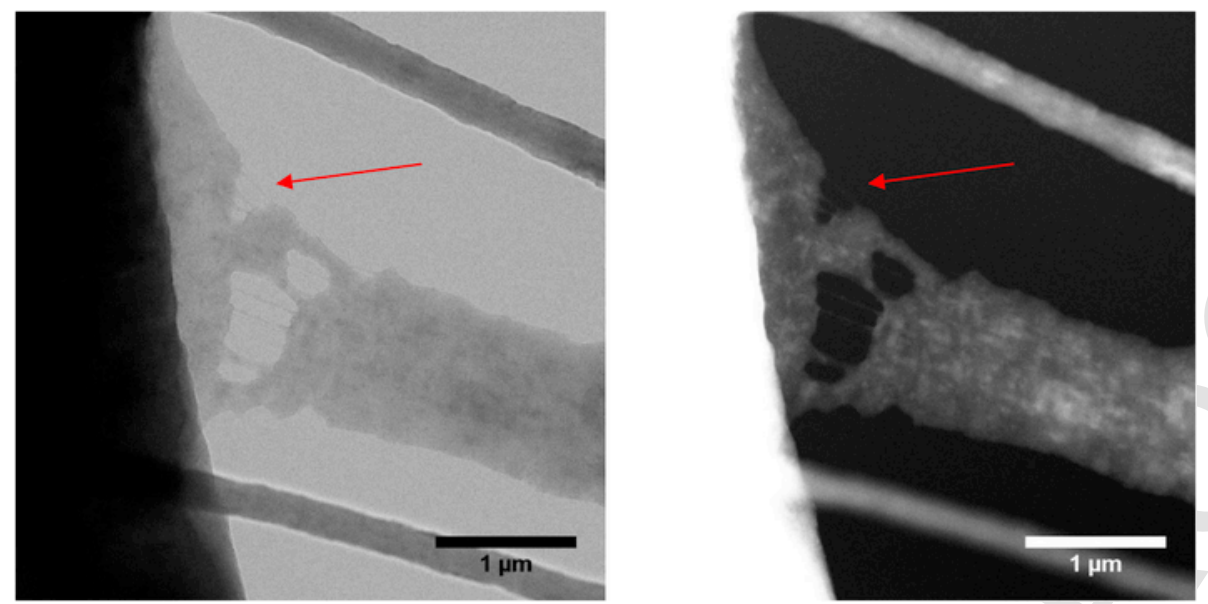

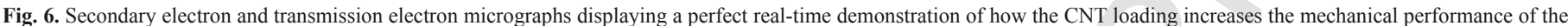
polymer nanofibers by bridging fractures and holding the fibre together. (A colour version of this figure can be viewed online.)
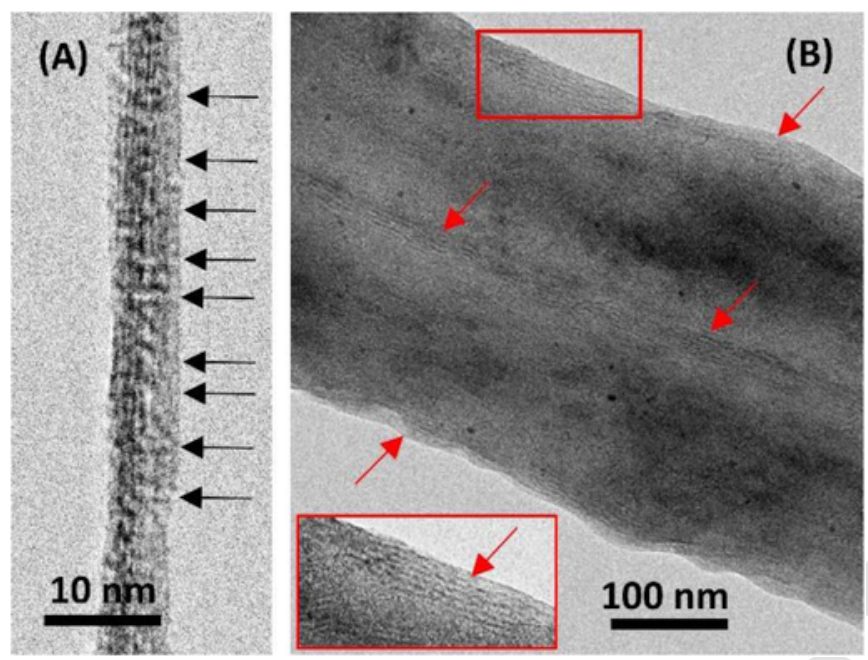

Fig. 7. Transmission electron micrographs displaying (A) arrowed shish-kebab crystalline structures along a CNT bundle within a PEO nanofiber, and (B) two larger adjoined nanofibers showing arrowed two-layer crystalline structures on the nanofiber surfaces. The boxed area is $2 \mathrm{x}$ zoom, highlighting the approximately $5 \mathrm{~nm}$ thick layers. (A colour version of this figure can be viewed online.)

electrostatic field, the stresses induce crystal growth as the nanofiber dries; as the nanofiber surface dries before the core, it is here where the bulk of the crystallisation can be found. To further explore the crystalline content of the nanofibers, DSC was employed to quantify and compare the crystallinity.

After acquisition of the DSC thermograms shown in Fig. 8, the percentage crystallinity of the as-electrospun nanofibers were calculated and tabulated in Table 1. It revealed that the samples containing CNT material had half the crystalline content of those tested without. Initially, this was thought to be a result of the CNT loading increasing the conductivity of the solution, which will reduce the static charging during electrospinning and the subsequent forces exerted on the fibre, thus reducing the level of stress-induced crystallisation $[3,42]$. However, calculating the percentage crystallinities from the second melt peaks, after each sample had recrystallised as a bulk material, revealed that crystalline content was still $50 \%$ lower for the samples with a CNT content. This confirmed that the introduction of the SDS coated nanotubes reduced the overall bulk crystallisation of the material, possibly by restricting chain movement or forming alternative structures, which does agree with other reports $[43,44]$. This is

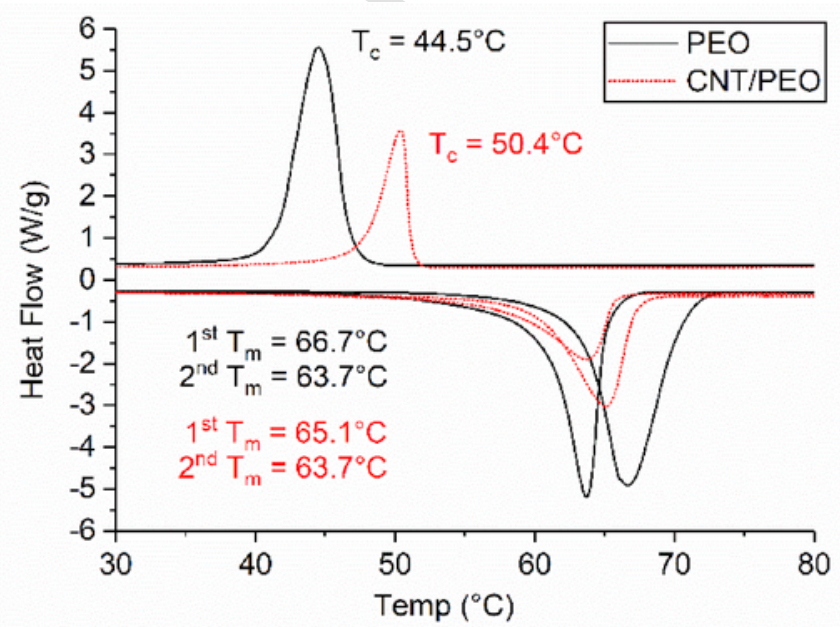

Fig. 8. DSC thermograms for the electrospun nanofibers comprised of PEO (black, solid) and CNT/PEO/SDS (red, dashed). For each sample the analysis was conducted in the order of heat-cool-heat, producing a 1 st melting peak $\left(1 \mathrm{st} \mathrm{T}_{\mathrm{m}}\right)$, a crystallisation peak $\left(\mathrm{T}_{\mathrm{c}}\right)$ and another melting peak ( $\left.2 \mathrm{nd} \mathrm{T}_{\mathrm{m}}\right)$. The CNT/PEO/SDS had a crystallinity of $32 \%$, significantly lower than the PEO sample with $62 \%$, but crystallised at a higher temperature. (A colour version of this figure can be viewed online.)

Table 1

The results from the DSC analysis, including the 1 st melting temperature $\left(1 \mathrm{st} \mathrm{T}_{\mathrm{m}}\right)$, the crystallisation temperature $\left(\mathrm{T}_{\mathrm{c}}\right)$, the calculated $\%$ crystallinity of the as-electrospun nanofibers, the 2 nd melting temperature $\left(2 n d \mathrm{~T}_{\mathrm{m}}\right.$ ) and the calculated $\%$ crystallinity of non-fibrous sample. The data reveals that the electrospinning process increases the crystallinity in the final nanofiber material.

\begin{tabular}{llllll}
\hline Sample & $\mathbf{1}^{\text {st }} \mathbf{T}_{\mathrm{m}}$ & $\mathbf{T}_{\mathrm{c}}$ & $\begin{array}{l}\text { Initial \% } \\
\text { Crystalline }\end{array}$ & $\mathbf{2}^{\text {nd }} \mathbf{T}_{\mathrm{m}}$ & $\begin{array}{l}\text { Final \% } \\
\text { Crystalline }\end{array}$ \\
\hline PEO & $66.7^{\circ} \mathrm{C}$ & $44.5^{\circ} \mathrm{C}$ & $62 \%$ & $63.7^{\circ} \mathrm{C}$ & $50 \%$ \\
CNT/PEO $/$ & $65.1^{\circ} \mathrm{C}$ & $50.4^{\circ} \mathrm{C}$ & $32 \%$ & $63.7^{\circ} \mathrm{C}$ & $22 \%$ \\
SDS & & & & & \\
\hline
\end{tabular}

counter-intuitive as a lower crystalline content is usually associated with poorer mechanical performances [45]. However, in our mechanical performance testing we found this to have the opposite effect for reasons explained in the following.

This result does not necessarily mean that SDS coated nanotubes prevent crystallisation, only that they restrict the natural preferred crystallisation growth mechanism of the PEO. When we compared 
the crystallisation temperature $\left(\mathrm{T}_{\mathrm{c}}\right)$ of each sample, we found that the samples with a CNT loading crystallise much earlier when cooling. This suggests that although the CNT presence reduces the overall crystallinity of the polymer, they equally promote smaller areas of crystallisation within their vicinity. Referring to back to Fig. 7, this crystallisation promotion would be the alternative 'shish-kebabs' nano-structures observed in the micrographs. This would also explain why other reports claim CNT incorporation promotes crystallisation, rather than reduce it $[46,47]$. This then means that the CNT alignment can lower crystalline content, but still improve mechanical performances by promoting a smaller specific crystal structures in an uni-directional manner along the axis of the fibers.

DSC analysis also confirms that the electrospinning process promotes crystallisation on the surface of the nanofibers, as shown in Fig. $7 \mathrm{~B}$. Comparing the percentage crystalline content between the initial as-electrospun nanofibers, before and after they have melted and cooled, revealed that the crystallinity reduces by approximately $10 \%$. This confirmed that approximately $10 \%$ of the crystal content in the nanofibers was induced during the electrospinning process.

To probe the microstructure of the nanofibers, SAXS analysis was conducted on CNT loaded nanofiber ropes which had not been subjected to any strain. Fig. 9 displays a typical representation of the diffraction pattern obtained during one step of the SAXS scan. The diffraction pattern obtained displays a strong diamond shape within the centre, elongated in the vertical direction; which suggests the presence of oriented voids or gaps along the fibers, which could be air or residual solvent [48]. In our case, these voids are likely due to either the space in between the tightly-rolled fibers or the space within the centre of the embedded carbon nanotubes.

In addition to the diamond shape in the centre of the diffraction pattern, intensity streaks are visible both above and below the centre at low q-values, indicating the presence of long range ordering (Fig. 9, image A). To analyse the data, a sector integral was applied to the region marked in Fig. 9, image B. The sector integral revealed several strong features that appear in each scanned step across the width of the nanofiber rope, giving several clues about the structure of the materials within the nanofibers. After the data was plotted, the three main peaks were re-plotted, removing the background reading obtained from the baseline; this then revealed another smaller peak (Peak 3 at $1.219 \mathrm{~nm}^{-1}$ ) (see Fig. 10).

Peaks 1 and 2 , corresponding to ' $\mathrm{d}$ ' spacings of $3.92 \mathrm{~nm}$ and $2.41 \mathrm{~nm}$, are a result of the X-ray scattering from the PEO/SDS [15]. Dror et al., whose data was included for a direct comparison, suggested that the peaks are the result of a two-layer lamella structure: a free SDS layer and an interacted PEO/SDS layer, leading to the long-range order shown by the two peaks. The ratio of these two peaks is close to $1 / \sqrt{ } 3$, which is associated with reflections of a hexagonal columnar structure of some nature [49-51]. This suggests that the
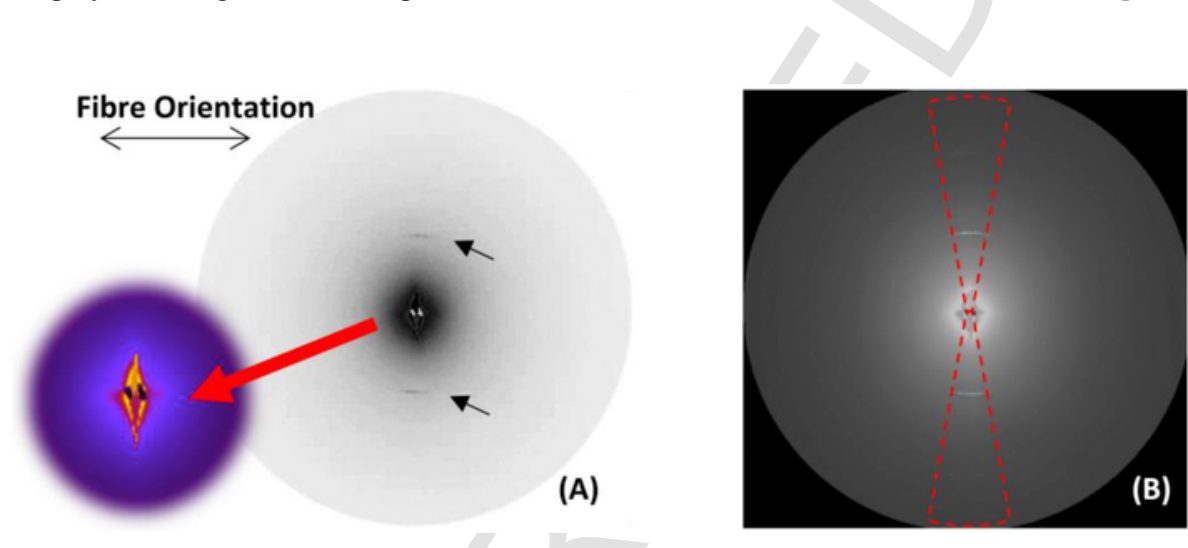

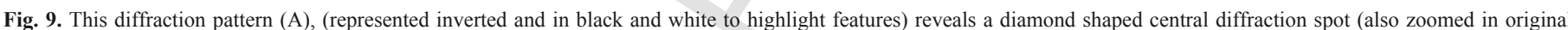

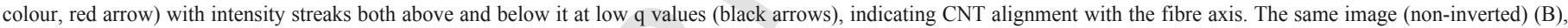
was then sector integrated over the red highlighted region as a function of $\mathrm{q}$ and plotted for analysis. (A colour version of this figure can be viewed online.)

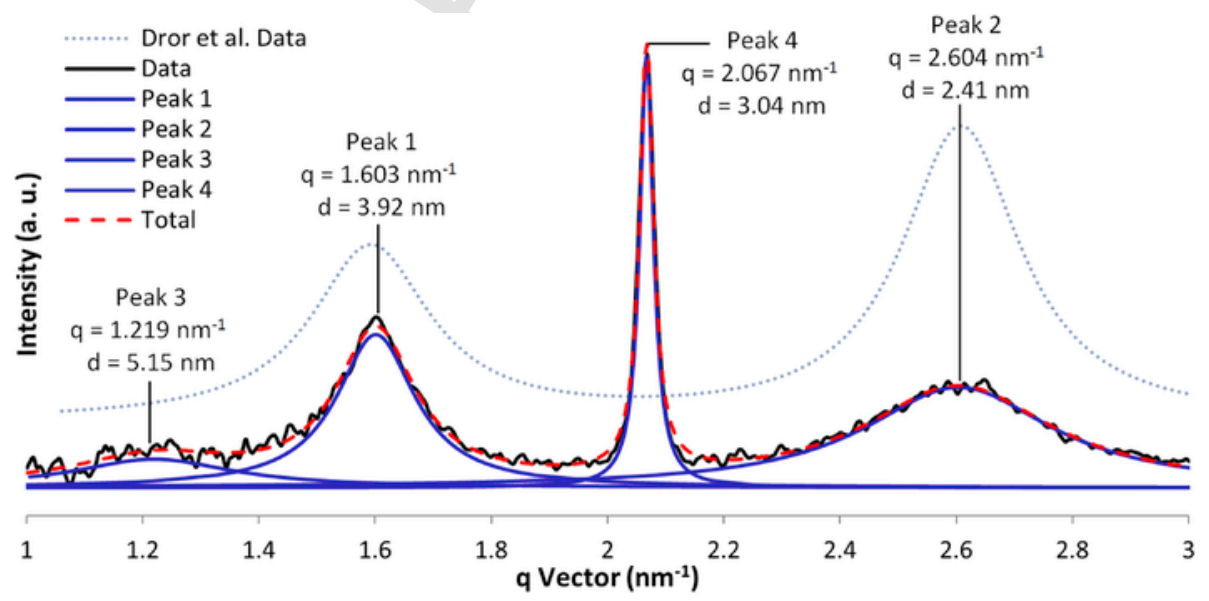

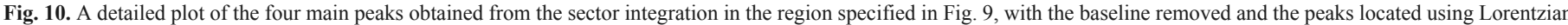

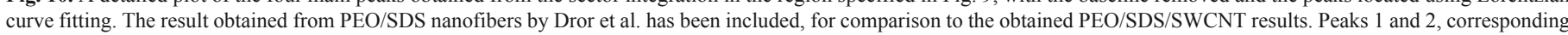

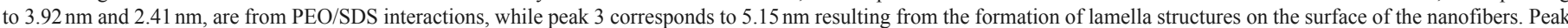

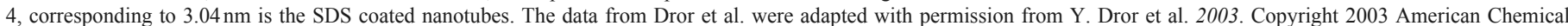
Society. (A colour version of this figure can be viewed online.) 
SDS/PEO forms a two-layer lamella structure but stacked as a hexagonal column [49]. Furthermore, these diffraction peaks were scattered perpendicular to the fibre orientation, indicating that these hexagonal columns have long-range along order parallel to the nanofiber orientation; i.e. they grow along the length of the nanofibers, as you would expect. Dror et al. then continued to explain that, after the CNT introduction, both peaks significantly broadened, suggesting that the CNT presence disrupts this purposed two-layer structure, which they attribute to the poor linearity of the CNT material they used. In our sample this was not the case, and both peaks remain relatively sharp, despite the CNT presence. This result is most likely due to using much higher quality of the nanotubes used, which are significantly more linear and do not inhibit directional crystal growth.

Peak 3 is of low intensity and just about visible through the signal noise, a Lorentzian fit shows the peak centre at $5.15 \mathrm{~nm}$. We attributed this to the two-layer lamella structures that we found in Fig. 7 on the surface of the nanofibers from the electrospinning process. It is possible that this peak could be a result of SDS micelles, however this was considered unlikely, as the sample tested contained a concentration of SDS significantly lower than the CMC $[15,52]$.

Peak 4 does not appear in Dror et al.'s data, indicating that this is a result of a regular structural change caused by the CNT presence, where literature confirms that this is the result of SDS wrapping of the nanotubes $[40,53,54]$. The structure factor has a d-spacing ratio of $1: 2$, a second peak at q-vector $4.148 \mathrm{~nm}^{-1}$, not shown in Fig. 10 for clarity but shown in Supplementary Information Fig. S2, further supports the conclusion that the nanotubes are wrapped in the SDS molecules in a lamellar arrangement [55-58]. Initially, when we first explored this signals origin, we considered that the d-spacing of this peak was too small for SDS coated nanotubes. However, Xin et al. studied this diffraction peak and found that it shifts to higher q-value as the ratio of polymer to SDS increases, indicating increased compact packing of the SDS molecules and resulting in a narrower d-spacing [53]. Lastly, we noted that this peak is much narrower, which indicates that the diffraction pattern is caused by a feature of specific and regular length scale, further supporting our claim that the CNT material used is of regular diameter, and high linearity.

\section{Conclusions}

The electrospinning process was successfully employed to manipulate carbon nanotubes into a highly aligned array within PEO, for use in advanced material composites. This alignment of the fibers and of the CNT content within the fibers were confirmed by electron microscopy and polarised Raman spectroscopy. Mechanical tensile testing demonstrated that a CNT loading of $3.9 \%$ increases the ultimate tensile strength by up to $320 \%$, the ductility by up to $315 \%$ and the Young's modulus by up to $380 \%$ (to $254 \mathrm{MPa}$ ). It was noted during testing that the appearance and feel of the fibers changed under stress, and with that transition electron microscopy, differential scanning calorimetry (DSC) and small angle X-ray scattering (SAXS) was used to probe the structure of the material. Evidence was found of nanofiber surface crystallisation from the electrospinning process, as well as the formation of 'shish-kebab' type growth along the length the CNT. DSC revealed that although the CNT content reduced the overall crystallinity of the material by $50 \%$, they also promote alternative crystal growth mechanisms, leading to an increased crystallisation temperature. SAXS confirmed that PEO was forming a two-layer hexagonal columnar structure with the SDS on the CNT surfaces. All of the crystalline changes induced by the CNT presence provided a 'ridged scaffold' for the polymer chains to bind to, preventing the chains from slipping during stress and leading to a significant mechanical enhancement observed during testing. Applications for this technology can include any material or device which requires specific crystalline unidirectional properties, where previously a stretching or drawing process would be needed during production to achieve the same result. A prime example is the need to pre-press polyacrylonitrile (PAN) to promote graphitisation during carbonisation in the manufacture of carbon fibers. Applying the research detailed here could potentially pre-align the PAN molecular chains, negating the need for a pre-stress process step before carbonisation and enhancing the performance of the final carbon fibers.

\section{Acknowledgment}

The authors would like to offer special thanks to Thomas Swan $\&$ Co. Ltd for the sponsorship of the research, and the EPSRC Centre for Doctoral Training at Surrey, MiNMaT programme, for funding and supporting this project (grant numbers EP/G037388/1 and EP/ N006372/1). The authors would also like to thank Violeta Doukova for her assistance with the DSC data acquisition, and the Diamond I22 Beamline team for their assistance with acquiring the SAXS data.

\section{Appendix A. Supplementary data}

Supplementary data related to this article can be found at https:// doi.org/10.1016/j.carbon.2018.06.019.

\section{References}

[1] M.F.L. De Volder, S.H. Tawfick, R.H. Baughman, A.J. Hart, Carbon nanotubes: present and future commercial applications, Sci. (New York, NY) 339 (6119) (2013) 535-539.

[2] R. Zhang, Y. Zhang, Q. Zhang, H. Xie, W. Qian, F. Wei, Growth of half-meter long carbon nanotubes based on Schulz-Flory distribution, ACS Nano 7 (7) (2013) 6156-6161.

[3] X. Sun, T. Chen, Z. Yang, H. Peng, The alignment of carbon nanotubes: an effective route to extend their excellent properties to macroscopic scale, Acc. Chem. Res. 46 (2) (2012) 539-549.

[4] A. Lekawa-Raus, T. Gizewski, J. Patmore, L. Kurzepa, K.K. Koziol, Electrical transport in carbon nanotube fibres, Scripta Mater. 131 (2017) 112-118.

[5] Y. Liang, D. Sias, P.J. Chen, S. Tawfick, Tough nano-architectured conductive textile made by capillary splicing of carbon nanotubes, Adv. Eng. Mater. (1600845) (2017) 1-7.

[6] J.N. Coleman, M. Cadek, K.P. Ryan, A. Fonseca, J.B. Nagy, W.J. Blau, M.S. Ferreira, Reinforcement of polymers with carbon nanotubes. The role of an ordered??polymer interfacial region. Experiment and modeling, Polymer 47 (26) (2006) 8556-8561

[7] X. Xie, Y. Mai, X. Zhou, Dispersion and alignment of carbon nanotubes in polymer matrix: a review, Mater. Sci. Eng. R Rep. 49 (4) (May 2005) 89-112.

[8] W.E. Teo, S. Ramakrishna, A review on electrospinning design and nanofibre assemblies, Nanotechnology 17 (no. 14) (Jul. 2006) R89-R106.

[9] S.G. King, L. McCafferty, V. Stolojan, S.R.P. Silva, Highly aligned arrays of super resilient carbon nanotubes by steam purification, Carbon N. Y. 84 (1) (2015) 130-137.

[10] S.G. King, V. Stolojan, S.R.P. Silva, Large area uniform electrospun polymer nanofibres by balancing of the electrostatic field, React. Funct. Polym. (October, 2017)

[11] C.Y. Li, L. Li, W. Cai, S.L. Kodjie, K.K. Tenneti, Nanohybrid shish-kebabs: periodically functionalized carbon nanotubes, Adv. Mater. 17 (9) (2005) 1198-1202.

[12] C. Boys, On the production, properties, and some suggested uses of the finest threads, Proc. Phys. Soc., London (1887) 8-19.

[13] H. Peng, Y. Liu, S. Ramakrishna, Recent development of centrifugal electrospinning, J. Appl. Polym. Sci. 134 (10) (2017) 1-10.

[14] H. Hou, J.J. Ge, J. Zeng, Q. Li, D.H. Reneker, A. Greiner, S.Z.D. Cheng, Electrospun polyacrylonitrile nanofibers containing a high concentration of well-aligned multiwall carbon nanotubes, Chem. Mater. 17 (5) (Mar. 2005) 967-973.

[15] Y. Dror, W. Salalha, R.L. Khalfin, Y. Cohen, A.L. Yarin, E. Zussman, Carbon nanotubes embedded in oriented polymer nanofibers by electrospinning, Langmuir 19 (17) (Aug. 2003) 7012-7020.

[16] W. Salalha, Y. Dror, R.L. Khalfin, Y. Cohen, A.L. Yarin, E. Zussman, Single-walled carbon nanotubes embedded in oriented polymeric nanofibers by electrospinning, Langmuir 20 (22) (Oct. 2004) 9852-9855. 
[17] Y. Song, Z. Sun, L. Xu, Z. Shao, Preparation and characterization of highly aligned carbon nanotubes/polyacrylonitrile composite nanofibers, Polymers 9 (1) (2017) 1-13.

[18] I. Dukovski, M. Muthukumar, Langevin dynamics simulations of early stage shish-kebab crystallization of polymers in extensional flow, J. Chem. Phys. 118 (14) (2003) 6648-6655.

[19] S. Liu, F. Zhang, G. Zheng, K. Dai, C. Liu, C. Shen, J.Z. Guo, Direct microscopic observation of shish-kebab structure in high-temperature electrospun iPP fibers, Mater. Lett. 172 (2016) 149-152.

[20] Y.-L. Li, I.A. Kinloch, M.S.P. Shaffer, J. Geng, B. Johnson, A.H. Windle, Synthesis of single-walled carbon nanotubes by a fluidized-bed method, Chem. Phys. Lett. 384 (no. 1-3) (Jan. 2004) 98-102.

[21] A.J. Blanch, C.E. Lenehan, J.S. Quinton, Optimizing surfactant concentration for dispersion of single-walled carbon nanotubes in aqueous solution, J. Phys. Chem. B 114 (30) (Aug. 2010) 9805-9811.

[22] M. Islam, E. Rojas, D. Bergey, A. Johnson, High weight fraction surfactant solubilization of single-wall carbon nanotubes in water, Nano Lett. 3 (2) (Feb. 2003) 269-273.

[23] I. Cotiuga, F. Picchioni, U.S. Agarwal, D. Wouters, J. Loos, P.J. Lemstra, Block-Copolymer-assisted solubilization of carbon nanotubes and exfoliation monitoring through viscosity, Macromol. Rapid Commun. 27 (13) (Jul. 2006) 1073-1078.

[24] Diamond Light Source, "I22-Small Angle Scattering - Diamond Light Source." [Online]. Available: http://www.diamond.ac.uk/Beamlines/ Soft-Condensed-Matter/small-angle/I22.html. [Accessed: 28-Feb-2018]

[25] B.B. Doyle, D.J.S. Hulmes, A. Miller, D.A.D. Parry, K.A. Piez, J. Woodhead-Galloway, A d-periodic narrow filament in collagen, Proc. R. Soc. Lond Ser. B Biol. Sci. 186 (1082) (May 1974) 67-74

[26] W. Folkhard, W. Geercken, E. Knörzer, E. Mosler, H. Nemetschek-Gansler, T. Nemetschek, M.H.J. Koch, Structural dynamic of native tendon collagen, J. Mol Biol. 193 (2) (Jan. 1987) 405-407.

[27] J. Hwang, H.H. Gommans, A. Ugawa, H. Tashiro, R. Haggenmueller, Polarized spectroscopy of aligned single-wall carbon nanotubes, Phys. Rev. B 62 (20) (2000) 4

[28] X. Wang, Z.Z. Yong, Q.W. Li, P.D. Bradford, W. Liu, D.S. Tucker, W. Cai, H. Wang, F.G. Yuan, Y.T. Zhu, Ultrastrong, stiff and multifunctional carbon nanotube composites, Mater. Res. Lett. 1 (1) (Oct. 2013) 1-7.

[29] R. Andrews, M. Weisenberger, Carbon nanotube polymer composites, Curr Opin. Solid State Mater. Sci. 8 (1) (Jan. 2004) 31-37.

[30] M.T. Byrne, Y.K. Gun'ko, Recent advances in research on carbon nanotube-polymer composites, Adv. Mater. 22 (15) (Apr. 2010) 1672-1688.

[31] L. Mandelkern, Crystallization of Polymers: Kinetics and Mechanisms, Cambridge University Press, 2004.

[32] C.H. Sherwood, F.P. Price, R.S. Stein, Effect of shear on the crystallization kinetics of poly(ethylene oxide) and poly(e-caprolactone) melts, J. Polym. Sci., Polym. Symp. 63 (1) (Mar. 1978) 77-94.

[33] K. Cui, L. Meng, Y. Ji, J. Li, S. Zhu, X. Li, N. Tian, D. Liu, L. Li, Extension-induced crystallization of poly(ethylene oxide) bidisperse blends: an entanglement network perspective, Macromolecules 47 (2) (2014) 677-686.

[34] B.-X. Yang, J.-H. Shi, K.P. Pramoda, S.H. Goh, Enhancement of stiffness, strength, ductility and toughness of poly(ethylene oxide) using phenoxy-grafted multiwalled carbon nanotubes, Nanotechnology 18 (12) (Mar. 2007) 125606.

[35] B. Kim, R. Porter, Uniaxial draw of poly (ethylene oxide) by solid-state extrusion, Macromolecules 18 (6) (1985) 1214-1217.

[36] H.Z. Geng, R. Rosen, B. Zheng, H. Shimoda, L. Fleming, J. Liu, O. Zhou, Fabrication and properties of composites of poly(ethylene oxide) and functionalized carbon nanotubes, Adv. Mater. 14 (19) (Oct. 2006) 1387-1390.

[37] E.P.S. Tan, C.N. Goh, C.H. Sow, C.T. Lim, Tensile test of a single nanofiber using an atomic force microscope tip, Appl. Phys. Lett. 86 (7) (2005) 073115

[38] L.M. Bellan, J. Kameoka, H.G. Craighead, Measurement of the Young's modul of individual polyethylene oxide and glass nanofibres, Nanotechnology 16 (8) (Aug. 2005) 1095-1099.

[39] L. Zhang, T. Tao, C. Li, Formation of polymer/carbon nanotubes nano-hybrid shish-kebab via non-isothermal crystallization, Polymer 50 (15) (2009) $3835-3840$
[40] L. Li, C.Y. Li, C. Ni, Polymer crystallization-driven, periodic patterning on carbon nanotubes, J. Am. Chem. Soc. 128 (5) (2006) 1692-1699.

[41] S. Prilutsky, E. Zussman, Y. Cohen, The effect of embedded carbon nanotubes on the morphological evolution during the carbonization of poly(acrylonitrile) nanofibers, Nanotechnology 19 (16) (2008).

[42] S. a. Theron, E. Zussman, A.L. Yarin, Experimental investigation of the governing parameters in the electrospinning of polymer solutions, Polymer 45 (6) (Mar. 2004) 2017-2030.

[43] T. Chatterjee, K. Yurekli, V.G. Hadjiev, R. Krishnamoorti, Single-walled carbon nanotube dispersions in poly(ethylene oxide), Adv. Funct. Mater. 15 (11) (Nov. 2005) 1832-1838.

[44] J. Jin, M. Song, F. Pan, A DSC study of effect of carbon nanotubes on crystallisation behaviour of poly(ethylene oxide), Thermochim. Acta 456 (1) (May 2007) 25-31.

[45] M.F. Talbott, G.S. Springer, L.A. Berglund, The effects of crystallinity on the mechanical properties of PEEK polymer and graphite fiber reinforced PEEK, J. Compos. Mater. 21 (11) (1987) 1056-1081.

[46] Y.-T. Shieh, G.-L. Liu, K.C. Hwang, C.-C. Chen, Crystallization, melting and morphology of PEO in PEO/MWNT-g-PMMA blends, Polymer 46 (24) (Nov. 2005) 10945-10951.

[47] R. Haggenmueller, J.E. Fischer, K.I. Winey, Single wall carbon nanotube/polyethylene nanocomposites: nucleating and templating polyethylene crystallites, Macromolecules 39 (2006) 2964-2971.

[48] J. Crawshaw, W. Bras, G.R. Mant, R.E. Cameron, Simultaneous SAXS and WAXS investigations of changes in native cellulose fiber microstructure on swelling in aqueous sodium hydroxide, J. Appl. Polym. Sci. 83 (6) (Feb. 2002) $1209-1218$.

[49] S. Laschat, A. Baro, N. Steinke, F. Giesselmann, C. Hägele, G. Scalia, R. Judele, E. Kapatsina, S. Sauer, A. Schreivogel, M. Tosoni, Discotic liquid crystals: from tailor-made synthesis to plastic electronics, Angew. Chem. Int. Ed. 46 (26) (2007) 4832-4887.

[50] G. Scalia, C. von Bühler, C. Hägele, S. Roth, F. Giesselmann, J.P.F. Lagerwall, Spontaneous macroscopic carbon nanotube alignment via colloidal suspension in hexagonal columnar lyotropic liquid crystals, Soft Matter 4 (3) (2008) 570.

[51] A. Pal, M.A. Kamal, V.A. Raghunathan, Observation of the chiral and achiral hexatic phases of self-assembled micellar polymers, Sci. Rep. 6 (2016) 1-8, July.

[52] D. Süss, Y. Cohen, Y. Talmon, The microstructure of the poly(ethylene oxide)/ sodium dodecyl sulfate system studied by cryogenic-temperature transmission electron microscopy and small-angle X-ray scattering, Polymer 36 (9) (Apr. 1995) 1809-1815.

[53] X. Xin, H. Li, E. Kalwarczyk, A. Kelm, M. Fiałkowski, E. Gorecka, D. Pociecha, R. Hołyst, Single-walled carbon nanotube/lyotropic liquid crystal hybrid materials fabricated by a phase separation method in the presence of polyelectrolyte, Langmuir 26 (11) (2010) 8821-8828.

[54] S. Dölle, E. Enz, G. Förster, J. Lagerwall, Liquid crystals from carbon nanotubes, Inst. Chem. (2010) 1-4.

[55] N. Stribeck, SAXS data analysis of a lamellar two-phase system. Layer statistics and compansion, Colloid Polym. Sci. 271 (11) (1993) 1007-1023.

[56] A. Wlochowicz, M. Eder, Distribution of lamella thicknesses in isothermally crystallized polypropylene and polyethylene by differential scanning calorimetry, Polymer 25 (9) (1984) 1268-1270.

[57] M.J. O'Connell, P. Boul, L.M. Ericson, C. Huffman, Y. Wang, E. Haroz, C. Kuper, J. Tour, K.D. Ausman, R.E. Smalley, Reversible water-solubilization of single-walled carbon nanotubes by polymer wrapping, Chem. Phys. Lett. 342 (no. 3-4) (Jul. 2001) 265-271.

[58] J.M. González-Domínguez, A. Ansón-Casaos, P. Castell, A.M. Díez-Pascual, M. Naffakh, G. Ellis, M. a. Gómez, M. Teresa Martínez, Integration of block copolymer-wrapped single-wall carbon nanotubes into a trifunctional epoxy resin. Influence on thermal performance, Polym. Degrad. Stabil. 95 (10) (Oct. 2010) 2065-2075. 